\title{
Saturation of Elliptic Flow and the Transport Opacity of the Gluon Plasma at RHIC
}

\author{
Dénes Molnár ${ }^{1,2}$ and Miklos Gyulassy ${ }^{1,2,3}$ \\ ${ }^{1}$ Physics Department, Columbia University, 538 W. 120th Street, New York, NY 10027 \\ ${ }^{2}$ RMKI Research Institute for Particle and Nuclear Physics, PO Box 49, H-1525 Budapest, Hungary \\ ${ }^{3}$ Collegium Budapest, Szentharomsag u. 2., H-1014 Budapest, Hungary
}

(Final published version + NPA703 Erratum)

\begin{abstract}
Differential elliptic flow and particle spectra are calculated taking into account the finite transport opacity of the gluon plasma produced in $\mathrm{Au}+\mathrm{Au}$ at $E_{c m} \sim 130 \mathrm{~A} \mathrm{GeV}$ at RHIC. Covariant numerical solutions of the ultrarelativistic Boltzmann equation are obtained using the MPC parton cascade technique. For typical pQCD $(\sim 3 \mathrm{mb})$ elastic cross sections, extreme initial gluon densities, $d N_{g} / d \eta \sim 15000$, are required to reproduce the elliptic flow saturation pattern reported by STAR. However, we show that the solutions depend mainly on the transport opacity, $\chi=\int d z \sigma_{t} \rho_{g}$, and thus the data can also be reproduced with $d N_{g} / d \eta \sim 1000$, but with extreme elastic parton cross sections, $\sim 45 \mathrm{mb}$. We demonstrate that the spectra and elliptic flow are dominated by numerical artifacts unless parton subdivisions $\sim 100-1000$ are applied to retain Lorentz covariance for RHIC initial conditions.
\end{abstract}

PACS 12.38.Mh;24.85.+p; 25.75.-q

\section{INTRODUCTION}

Differential elliptic flow, $v_{2}\left(p_{\perp}\right)=\langle\cos (2 \phi)\rangle_{p_{\perp}}$, the second Fourier moment of the azimuthal momentum distribution for fixed $p_{\perp}$, is one of the important experimental probes of collective dynamics in $A+A$ reactions [1-13]. The discovery [14] of a factor of two enhancement of elliptic flow in noncentral nuclear collisions at RHIC relative to SPS [15] has generated even more interest in this "barometric" measure of collective transverse flow. In addition, preliminary STAR data reported in [16] suggest a remarkable saturation property of this flow at high $p_{\perp}$ with $v_{2}\left(p_{\perp}>2 \mathrm{GeV}\right) \sim 0.15$. This corresponds to a factor of two azimuthal angle asymmetry of high- $p_{\perp}$ particle production relative to the reaction plane. This collective effect depends strongly on the dynamics in a heavy ion collision and therefore provides important constraints about the density and effective energy loss of partons. In particular, we show that it constrains the transport opacity of the produced gluon plasma.

Predictions of collective elliptic flow in noncentral nuclear collisions were first based on ideal (nondissipative) hydrodynamics $[1,2,6]$. Unlike at lower energies, ideal hydrodynamics seems to reproduce the (low $p_{T}<2 \mathrm{GeV}$ ) data [14] at RHIC remarkably well. However, it fails to saturate at high $p_{\perp}>2 \mathrm{GeV}$ as indicated by the preliminary data [16]. The hydrodynamic results were found in [6] to be surprisingly insensitive to the choice of ini- tial conditions, equation of state and freezeout criteria, once the observed $d N_{c h} / d \eta$ was reproduced, leaving no adjustable hydrodynamic model parameters with which the saturation property could be reproduced.

The lack of saturation in ideal hydrodynamics is due to the assumption of zero mean free path and that local equilibrium can be maintained until a chosen freezeout $3 \mathrm{D}$ hypersurface is reached. This idealization is certainly invalid outside some finite domain of phase space in heavy ion collisions [17]. Finite transition rates are expected to produce nonequilibrium deviations from the predicted hydrodynamic flow pattern. Covariant Boltzmann transport theory provides a convenient framework to estimate dissipative effects. The assumption of local equilibrium is replaced by the assumption of a finite local mean free path $\lambda(s, x) \equiv 1 / \sigma(s) n(x)$. The theory then naturally interpolates between free streaming $(\lambda=\infty)$ and ideal hydrodynamics $(\lambda=0)$.

The previous calculations of collective flow from covariant transport theory $[9,18,19]$ lead to too small collective effects. This was due to the use of small perturbative QCD cross sections and dilute parton initial densities based on HIJING [20]. Recently, denser parton initial conditions were suggested based on gluon saturation models [21]. Initial gluon densities up to five times higher than from HIJING were predicted. The question studied in this paper is whether such initial conditions may be dense enough to generate the observed collective flow even with pQCD elastic cross sections. In this paper, we explore the dependence of differential elliptic flow on initial conditions, or equivalently ${ }^{1}$, on the magnitude of partonic cross section.

We note that most hadronic cascade models supplemented with string dynamics $[4,11]$ underpredict elliptic flow because the spatial asymmetry is too small after hadronization to generate the observed momentum space asymmetry. The reason is that hadronization through longitudinal string excitations reduces the strength of partonic level elliptic flow. To produce sufficient elliptic flow in string models, other mechanisms have to be included such as color exchange. While the impact parameter dependence of elliptic flow at RHIC can be repro-

\footnotetext{
${ }^{1}$ The equivalence is due to the scaling property explained in Section II B.
} 
duced via color exchange [10], it is not known whether the saturation property studied here can also be explained.

The saturation and eventual decrease of $v_{2}$ at high $p_{\perp}$ has been predicted as a consequence of finite inelastic parton energy loss $[12,13]$. Those predictions however assumed the validity of an Eikonal approach at moderate $p_{T} \sim 10 \mathrm{GeV}$. In addition, the pQCD computable jet quenched part had to be joined phenomenologically onto a parametrized "soft" nonperturbative component below $p_{T}<2 \mathrm{GeV} / \mathrm{c}$. Covariant transport theory overcomes the need to treat soft and hard dynamics on different footings. It is the only practical self-consistent theoretical tool at present to address simultaneously both the soft collective component and the far from equilibrium high$p_{\perp}$ component. While current parton cascade techniques lack at present a practical means to implement covariant inelastic energy loss, it is of considerable theoretical interest to solve covariant Boltzmann theory even in the elastic limit since so few solutions are known. We solve that theory numerically here to get insight into the dynamical interplay between the soft and hard components over a wide dynamical range of parameters.

For large enough elastic opacities, the observed collective flow strength can certainly be reproduced [19]. The outstanding question which we focus on is whether the detailed pattern of deviations from ideal hydrodynamic flow and its saturation at high $p_{\perp}$ can also be understood quantitatively in this particular dynamical framework.

Forerunners of this study $[9,19]$ computed elliptic flow for partonic systems starting from initial conditions expected at RHIC. In this paper we extend those studies in three aspects. We compute the $p_{\perp}$-differential elliptic flow $v_{2}\left(p_{\perp}\right)$. The consequences of hadronization are investigated, which is necessary to compare to the observations. Finally, we use realistic diffuse nuclear geometry for the initial conditions.

We compute the partonic evolution with MPC [22], a newly formulated, covariant, parton kinetic theory technique. $\mathrm{MPC}$ is an extension of the covariant parton cascade algorithm, ZPC [23]. Both MPC and ZPC have been extensively tested $[24,25]$ and compared to analytic transport solutions and covariant Euler and Navier-Stokes dynamics in $1+1 \mathrm{D}$ geometry. A critical feature of both these algorithms is the implementation of the parton subdivision technique proposed by Pang $[25,26]$.

Extensions of MPC to include inelastic $2 \leftrightarrow 3$ partonic processes [18] are under development. In this paper, we apply MPC in the pure elastic parton interactions mode as in ZPC [27].

\section{COVARIANT TRANSPORT THEORY}

\section{A. Transport equation}

We consider here, as in Refs. [26,23,22,17], the simplest but nonlinear form of Lorentz-covariant Boltzmann transport theory in which the on-shell phase space density $f(x, \mathbf{p})$, evolves with an elastic $2 \rightarrow 2$ rate as

$$
\begin{aligned}
p_{1}^{\mu} \partial_{\mu} f_{1}= & \iiint_{234}\left(f_{3} f_{4}-f_{1} f_{2}\right) W_{12 \rightarrow 34} \delta^{4}\left(p_{1}+p_{2}-p_{3}-p_{4}\right) \\
& +S\left(x, \mathbf{p}_{1}\right) .
\end{aligned}
$$

Here $W$ is the square of the scattering matrix element, the integrals are shorthands for $\int_{i} \equiv \int \frac{g d^{3} p_{i}}{(2 \pi)^{3} E_{i}}$, where $g$ is the number of internal degrees of freedom, while $f_{j} \equiv f\left(x, \mathbf{p}_{j}\right)$. The initial conditions are specified by the source function $S(x, \mathbf{p})$, which we discuss at the end of this Subsection.

For our applications below, we interpret $f(x, \mathbf{p})$ as describing an ultrarelativistic massless gluon gas with $g=16$ (8 colors, 2 helicities). We neglect quark degrees of freedom because at RHIC gluons are more abundant.

In principle, the transport equation (1) could be extended for bosons with the substitution $f_{1} f_{2} \rightarrow f_{1} f_{2}(1+$ $\left.f_{3}\right)\left(1+f_{4}\right)$ and a similar one for $f_{3} f_{4}$. In practice, no covariant algorithm yet exists to handle such nonlinearities. We therefore limit our study to quadratic dependence of the collision integral on $f$.

The elastic gluon scattering matrix elements in dense parton systems are modeled by a Debye-screened form as in Ref. [9]:

$$
\frac{d \sigma_{e l}}{d t}=\sigma_{0}(s)\left(1+\frac{\mu^{2}}{s}\right) \frac{\mu^{2}}{\left(t+\mu^{2}\right)^{2}},
$$

where $\mu$ is the screening mass, $\sigma_{0}(s)=9 \pi \alpha_{s}^{2}(s) / 2 \mu^{2}$ is the total cross section. For simplicity, we will assume $\sigma_{0}$ to be energy independent, neglecting its weak logarithmic dependence on $s$ in the energy range relevant at RHIC.

For small values of $\mu$, forward-peaked scattering is favored, while as $\mu$ increases the angular distribution becomes more and more isotropic. For a fixed total cross section $^{2}$, the relevant transport cross section is

$$
\begin{aligned}
\sigma_{t}(s) & \equiv \int d \sigma_{e l} \sin ^{2} \theta_{c m}=\int d t \frac{d \sigma_{e l}}{d t} \frac{4 t}{s}\left(1-\frac{t}{s}\right) \\
& =\sigma_{0} 4 z(1+z)[(2 z+1) \ln (1+1 / z)-2]
\end{aligned}
$$

where $z \equiv \mu^{2} / s$. This is a monotonic function of $\mu$ and maximal in the isotropic $(\mu \rightarrow \infty)$ case. In the small angle dominated limit, with $z \ll 1, \sigma_{t} / \sigma_{0} \approx 4 z(\ln 1 / z-$ $2)$.

It is important to emphasize that while the cross section suggests a geometrical picture of action over finite distances, we use Eq. (2) only as a convenient parametrization to describe the effective local transition

\footnotetext{
${ }^{2}$ To keep the total cross section constant as a function of $\mu$, one of course has to adjust the coupling $\alpha_{s}$ accordingly.
} 
probability, $W$. In the present study this is simply modeled as $W(t)=s d \sigma / d t$. The particle subdivision technique (see next Subsection) needed to recover covariance removes all notion of nonlocality in this approach, just like in hydrodynamics. Thus, the cross sections, e.g., 100 mb, used in the present study to simulate a high collision rate do not imply acausal action at a distance.

For $\mathrm{Au}+\mathrm{Au}$ collisions, the initial condition was taken to be a longitudinally boost invariant Bjorken tube in local thermal equilibrium at temperature $T_{0}$ at proper time $\tau_{0}=0.1 \mathrm{fm} / c$ with uniform pseudorapidity $\eta \equiv$ $1 / 2 \log ((t+z) /(t-z))$ distribution between $|\eta|<5$. The transverse density distribution was assumed to be proportional to the binary collision distribution for two Woods-Saxon distributions. For collisions at impact parameter $b$ the transverse binary collision profile is

$$
\frac{d N(\mathbf{b})}{d \eta d^{2} \mathbf{x}_{\perp}}=\sigma_{j e t} T_{A}\left(\mathbf{x}_{\perp}+\frac{\mathbf{b}}{2}\right) T_{A}\left(\mathbf{x}_{\perp}-\frac{\mathbf{b}}{2}\right)
$$

where $T_{A}(\mathbf{b}) \equiv \int d z \rho_{A}\left(\sqrt{z^{2}+\mathbf{b}^{2}}\right)$, in terms of the diffuse nuclear density $\rho_{A}(r)$. The pQCD jet cross section normalization, $\sigma_{j e t}$, and the temperature $T_{0}$ were determined by fitting the gluon minijet transverse momentum spectrum predicted by HIJING [20] for central $\mathrm{Au}+\mathrm{Au}$ collisions at $\sqrt{s}=130 \mathrm{~A} \mathrm{GeV}$ (without shadowing and jet quenching). This gives $d N(0) / d \eta=210$ and $T_{0}=700$ $\mathrm{MeV}$.

Evolutions from different initial densities (but the same density profile) can be obtained by varying the cross section only and using the scaling property explained in Section II C.

\section{B. Parton Subdivision}

We utilize the parton cascade method to solve the Boltzmann transport equation (1). A critical drawback of all cascade algorithms is that they inevitably lead to numerical artifacts that violate Lorentz covariance. This occurs because particle interactions are simulated to occur whenever the distance of closest approach (in the relative c.m.) is $d<\sqrt{\sigma_{0} / \pi}$.

Acausal (superluminal) propagation due to action at a distance leads to severe numerical artifacts. In particular, the transverse energy evolution $d E_{T}(\tau) / d y$ and the final asymptotic transverse energy per unit rapidity are frame dependent [17].

To recover the local character of equation (1) and hence Lorentz covariance, it is essential to use the parton subdivision technique $[26,23]$. This technique is based on the covariance of Eq. (1) under the transformation

$$
f \rightarrow f^{\prime} \equiv \ell f, \quad W \rightarrow W^{\prime} \equiv W / \ell \quad\left(\sigma \rightarrow \sigma^{\prime}=\sigma / \ell\right),
$$

where $\ell$ is the number of particle subdivisions. The magnitude of numerical artifacts is governed by the diluteness of the system $\sqrt{\sigma} / \lambda_{M F P}$, which scales with $1 / \sqrt{\ell}$
[25]. Lorentz violation therefore formally vanishes in the $\ell \rightarrow \infty$ limit. The convergence to the accurate covariant solution with $\ell$ is slower if the density or cross section increases.

Figures 1 and 2 illustrate the effect of Lorentz violation on the observables studied in this paper. For insufficient particle subdivision, elliptic flow and the $p_{\perp}$ spectra are dominated by numerical artifacts. In particular, elliptic flow is significantly underpredicted, while the high$p_{\perp}$ spectrum exhibits an unphysical "reheating" during the expansion. For the initial conditions for these plots, these numerical artifacts disappear only when the particle subdivision reaches $\sim 200$. This reinforces the results by Ref. [17], where very high $\sim 100-1000$ subdivisions were needed to obtain accurate numerical solutions of the transport equation (1) for initial conditions expected at RHIC.

\section{Scaling of the transport solutions}

Subdivision covariance (5) actually implies that the transport equation has a broad dynamical range, and the solution for any given initial condition and transport property immediately provides the solution to a broad band of suitably scaled initial conditions and transport properties. This is because solutions for problems with $\ell$ times larger the initial density $d N / d \eta d^{2} x_{\perp}$, but with one $\ell$-th the reaction rate can be mapped to the original $(\ell=1)$ case for any $\ell$. We must use subdivision to eliminate numerical artifacts. However, once that is achieved, we have actually found the solution to a whole class of suitably rescaled problems.

The dynamical range of the transport equation (1) is further increased by its covariance under coordinate and momentum rescaling [17], leading to covariance of the transport theory under

$$
\begin{aligned}
f(x, \mathbf{p}) & \rightarrow f^{\prime}(x, \mathbf{p}) \equiv \ell_{p}^{-3} \ell f\left(\frac{x}{\ell_{x}}, \frac{\mathbf{p}}{\ell_{p}}\right), \\
W\left(\left\{p_{i}\right\}\right) & \rightarrow W^{\prime}\left(\left\{p_{i}\right\}\right) \equiv \frac{\ell_{p}^{2}}{\ell_{x} \ell} W\left(\left\{\frac{p_{i}}{\ell_{p}}\right\}\right), \\
m & \rightarrow m^{\prime}=m / \ell_{p},
\end{aligned}
$$

where $\ell_{x}$ and $\ell_{p}$ are the coordinate and momentum scaling parameters, respectively. This means [17] that we can scale one solution to others provided that both $\mu / T_{0}$ and $\sigma_{0} d N / d \eta$ remain the same (we cannot exploit coordinate scaling because the nuclear geometry is fixed). For example, three times the density with one-third the cross section leaves both parameters the same, hence the results can be obtained via scaling without further computation.

In general the numerical (cascade) solution of Eq. (1) tends in the $\ell \rightarrow \infty$ limit toward a covariant physical solution that depends on two scales, $\mu / T_{0}$ and $\sigma_{0} d N / d \eta$. In 
an Eikonal picture of high- $p_{\perp}$ production, the distributions are expected to depend on the opacity or the mean number of collisions in the medium

$$
\begin{aligned}
\langle n\rangle & =\frac{L}{\lambda_{e l}}=\int d t \frac{d \sigma_{e l}}{d t} \int d z \rho\left(\mathbf{x}_{0}+z \hat{\mathbf{n}}, \tau=\frac{z}{c}\right) \\
& \approx \frac{d N}{d y} \frac{\sigma_{0}}{2 \pi R_{G}^{2}} \log \frac{R_{G}}{\tau_{0}},
\end{aligned}
$$

where $\tau_{0}$ is the formation proper time and $R_{G}$ is the effective Gaussian transverse coordinate rms radius.

Our numerical results in Table I show that for a given centrality and initial gluon density, the average number of collisions per parton is within $10 \%$ accuracy proportional to $\sigma_{0}$ and does not depend on $\mu / T_{0}$ :

$$
\left\langle n\left(b, \sigma_{0} \frac{d N(0)}{d \eta}, \frac{\mu}{T_{0}}\right)\right\rangle \approx \frac{\sigma_{0}}{\sigma_{0}^{\prime}}\left\langle n\left(b, \sigma_{0}^{\prime} \frac{d N(0)}{d \eta}, \frac{\mu^{\prime}}{T_{0}^{\prime}}\right)\right\rangle .
$$

Therefore, one would naively expect $\sigma_{0} d N / d \eta$ to be the relevant scale.

However, from the point of view of dissipative dynamics via Navier-Stokes and Fokker-Planck equation, the more relevant dynamical parameter is the effective transport opacity

$$
\chi \equiv \frac{\sigma_{t}}{\sigma_{0}}\langle n\rangle=\sigma_{t}\left\langle\int d z \rho\left(\mathbf{x}_{0}+z \hat{\mathbf{n}}, \tau=\frac{z}{c}\right)\right\rangle .
$$

The ensemble average over initial coordinates and directions is implied above. In addition, $\sigma_{t}$ here stands for $\sigma_{t} \equiv \sigma_{t}(\langle s\rangle)$, where $\langle s\rangle=18 T_{0}^{2}$ is the initial thermal average of $s$. This is an approximation to the ensemble average $\left\langle\sigma_{t}(s)\right\rangle$.

In general, the transport opacity is a dynamical quantity that we do not know until we solved the transport equation for the set of parameters $b, \sigma_{0} d N(0) / d \eta$, and $\sigma_{t} / \sigma_{0}$ (or equivalently, $\mu / T_{0}$ ). However, Eqs. (8) and (9) imply that for the range of parameters considered in this study it is approximately proportional to the product of the two scales $\sigma_{0} d N(0) / d \eta$ and $\sigma_{t} / \sigma_{0}$ :

$$
\chi\left(b, \sigma_{0} \frac{d N(0)}{d \eta}, \frac{\sigma_{t}}{\sigma_{0}}\right) \approx \frac{\sigma_{t} \frac{d N(0)}{d \eta}}{\sigma_{0}^{\prime} \frac{d N^{\prime}(0)}{d \eta}}\left\langle n\left(b, \sigma_{0}^{\prime} \frac{d N^{\prime}(0)}{d \eta}\right)\right\rangle .
$$

The nontrivial, impact parameter dependent part $\left\langle n\left(b, \sigma_{0} d N(0) / d \eta\right\rangle\right.$ is the average number of collisions per parton as a function of $b$ for a fixed value of $\sigma_{0} d N(0) / d \eta$ (and an arbitrary $\mu / T_{0}$ ), which is tabulated in Table I. For example, one could use the values of set E), in which case the corresponding proportionality constant is $\sigma_{0}^{\prime} d N^{\prime}(0) / d \eta=3 \mathrm{mb} \times 210=630 \mathrm{mb}$.

Of course, there is no a priori guarantee that the solutions of Eq. (1) depend only on this transport opacity parameter. However, as demonstrated in Fig. 3, this turns out to hold within $\sim 10-20 \%$ accuracy for elliptic flow and the transverse momentum spectra out to 6 $\mathrm{GeV} / \mathrm{c}$ for the parameters and initial conditions appropriate at RHIC energies. In particular, simulations with very different impact parameters give the same ratio between the initial and final spectrum, provided $\chi$ is the same. Hence, the relevant parameter that governs the evolution of the $p_{\perp}$ spectra is $\chi$ alone. This is not so for elliptic flow, which is also driven by the initial spatial anisotropy and thus depends not only on $\chi$ but also on the impact parameter.

\section{NUMERICAL RESULTS FOR THE PARTONIC EVOLUTION}

In this Section we present elliptic flow results and $p_{\perp}$ spectra for the partonic evolution.

Under the scaling (6) the differential elliptic flow $v_{2}\left(p_{\perp}\right)$ and the $p_{\perp}$ spectrum transform as

$$
\begin{gathered}
v_{2}\left(p_{\perp}\right) \rightarrow v_{2}^{\prime}\left(p_{\perp}\right) \equiv v_{2}\left(\frac{p_{\perp}}{\ell_{p}}\right) \\
\frac{d N}{d^{2} p_{\perp}}\left(\mathbf{p}_{\perp}\right) \rightarrow \frac{d N^{\prime}}{d^{2} p_{\perp}}\left(\mathbf{p}_{\perp}\right)=\ell \frac{d N}{d^{2} p_{\perp}}\left(\frac{\mathbf{p}_{\perp}}{\ell_{p}}\right) .
\end{gathered}
$$

Hence elliptic flow depends on $\sigma_{t}$ and $d N / d \eta$ only through the product $\sigma_{t} d N / d \eta$. On the other hand, the $p_{\perp}$ spectrum depends on $\sigma_{t}$ and $d N / d \eta$ separately.

As emphasized in the previous Section, we will label the results by the effective elastic transport opacity $\chi$ from Eq. (9) and the impact parameter $b$. Possible initial gluon densities and transport cross sections corresponding to a given transport opacity $\chi$ and impact parameter $b$ can be extracted using Eq. (10), while possible cutoffs and total cross sections corresponding to a given transport cross section can be obtained from Eq. (3). These mappings are not unique. A given $\chi$ and $b$ correspond to a whole class of possible initial densities, total cross sections and cutoffs.

Table I shows the set of simulation parameters for each simulation, together with $\chi$ determined directly from the average number of cascade collisions per particle. In Table I we also introduced letter codes A) through F) as a quick reference to particular subsets of simulation parameters. We will include this letter code on most labels together with $\chi$, for convenience.

The evolution was performed numerically with 40 and $100 \mathrm{mb}$ isotropic cross sections, and with 3, 40 and 100 mb gluonic cross sections with $\mu / T_{0}=1$. We used particle subdivision $\ell=100$ for impact parameters 0,2 , and $4 \mathrm{fm}$, while $\ell=220,450,1100$, and 5000, for $b=6$, 8,10 , and $12 \mathrm{fm}$. Our study of subdivision convergence shown in Figs. 1 and 2 indicates that with $b=8 \mathrm{fm}$ and $\chi=9.74^{A}$ ) a particle subdivision of $\ell \sim 200-250$ is required, which means that for $b=0,2$, and $4 \mathrm{fm}$, $\ell=100$ is not sufficient if $\chi>8-10$. Unfortunately, our computational resources were insufficient to allow higher subdivision runs. While this affects elliptic flow results little because most elliptic flow contributions come from $b>4 \mathrm{fm}$, the particle spectra are affected significantly. Therefore, we only present spectra for $b \geq 6 \mathrm{fm}$. 


\section{A. Elliptic flow results}

Figures 4 and 5 show the final asymptotic gluon elliptic flow as a function of transverse momentum for different impact parameters with $\left.\sigma_{t} d N(0) / d \eta \approx 2580^{C}\right) \mathrm{mb}$ and $\left.6440^{A}\right) \mathrm{mb}$, respectively (the corresponding transport opacities at $b=0$ are $\left.\chi_{b=0}=7.90^{C}\right)$ and $\left.19.4^{A)}\right)$. With increasing $p_{\perp}$, elliptic flow increases until $p_{\perp} \sim 1.5-2$ $\mathrm{GeV}$, where it saturates, reproducing the pattern observed at RHIC $[14,16]$. With increasing impact parameter, elliptic flow first monotonically increases, then monotonically decreases, showing a maximum at $b \approx 8 \mathrm{fm}$. These features were universal for all the cross sections we studied, except for a small increase in the location of the maximum with increasing transport cross section, as can be seen in Fig. 6, from $b=7 \mathrm{fm}$ at $\sigma_{t}=0.91^{E}$ ) $\mathrm{mb}$ to $9 \mathrm{fm}$ at $66^{B}$ ) $\mathrm{mb}$. Also, as expected, elliptic flow is a monotonically increasing function of the transport opacity, if the impact parameter is kept fixed.

Figure 6 shows the $p_{\perp}$-integrated gluon elliptic flow as a function of centrality. The cascade reproduces the trend seen in the STAR data [14] down to very small centralities $\sim 0.1-0.2$, where the ideal hydrodynamical assumption of zero mean free path certainly breaks down. To quantitatively reproduce the data, transport opacities $\chi_{b=0} \sim 8-14$ are needed. With the pQCD elastic $g g$ cross section $\sigma_{0}(\mu=T) \approx 3 \mathrm{mb}$, this corresponds to an initial gluon density $d N_{g}(0) / d \eta \sim 3000-5000$.

Figure 7 shows the impact-parameter-averaged gluon elliptic flow as a function of transverse momentum for different transport opacities. The impact-parameteraveraged flow was computed via the formula

$$
v_{2}^{a v}\left(p_{\perp}\right) \equiv \frac{2 \pi}{\pi b_{\max }^{2}} \int_{0}^{b_{\max }} d b b \frac{\int d \phi \cos (2 \phi) \frac{d N}{d \eta d^{2} p_{\perp}}(b)}{\int d \phi \frac{d N}{d \eta d^{2} p_{\perp}}(b)}
$$

with $b_{\max }=12 \mathrm{fm}$. As we show in the Appendix, for our transport theory solutions, Eq. (12) gives comparable results to the minimum-bias differential elliptic flow defined by STAR as

$$
v_{2}^{S T A R}\left(p_{\perp}\right) \equiv \frac{\int_{0}^{b_{\max }} b d b \int d \phi \cos (2 \phi) \frac{d N}{d \eta d^{2} p_{\perp}}(b)}{\int_{0}^{b_{\max }} b d b \int d \phi \frac{d N}{d \eta d^{2} p_{\perp}}(b)}
$$

which weights flow in more central events preferentially. This is not the case for ideal hydrodynamic solutions [6], for which the STAR definition [14] results in much smaller flow than if Eq. (12) is used.

We use the definition (12) because it can be computed numerically more reliably in our approach. As discussed in the beginning of Section III, it is difficult to reduce cascade numerical artifacts to an acceptable level when the transport opacity $\chi$ is large. For all other parameters kept fixed, $\chi$ increases with decreasing impact parameter, hence the STAR definition that weights $v_{2}$ at small $b$ preferentially is more prone to such numerical artifacts.
Varying the magnitude of energy loss we searched for the drop in $v_{2}\left(p_{\perp}\right)$ at high $p_{\perp}$ predicted by calculations based on inelastic parton energy loss $[12,13]$. Although those studies consider only effects due to radiative energy loss, one expects a similar behavior in case of purely elastic energy loss.

Figure 8 shows the dependence of $v_{2}\left(p_{\perp}\right)$ on the transport opacity for a fixed impact parameter. We varied the opacity by changing the screening mass $\mu$. As expected, elliptic flow decreases with decreasing $\chi$. However, there is no sign of a drop at high $p_{\perp}$ : within statistical errors, the results are consistent with a constant flow from 2 to $6 \mathrm{GeV}$ transverse momentum.

\section{B. Particle spectra}

Figures 9-11 show the final gluon $p_{\perp}$ spectra from MPC as a function of transport opacity and impact parameter. To show more clearly the degree of quenching due to multiple elastic collisions, we plot in Figs. 9 and 10 the ratio of the final spectra to the initial for $b=6,8,10$, and $12 \mathrm{fm}$. With HIJING initial densities and pQCD elastic cross sections, $\chi \sim 0.02-0.2$ is too small to produce more than $\sim 10 \%$ quenching. As we increase the transport opacity, quenching of the $p_{\perp}>2 \mathrm{GeV} / \mathrm{c}$ range increases and by $\chi \sim 14-16$ it reaches a factor of ten suppression at $p_{\perp}>6 \mathrm{GeV}$.

While the quenching depends on $\chi$ only, the absolute yield is proportional to the initial $d N / d \eta$. Hence from the absolutely normalized measured spectrum at a given centrality one could extract both $\sigma_{t}$ and $d N(0) / d \eta$. As seen in Fig. 11, the quenching at high $p_{\perp}$ is complemented by an enhancement at low $p_{\perp}$. For clarity, we normalized all curves at $p_{\perp}=2 \mathrm{GeV}$. While the STAR data [30] are too preliminary to show yet, the slope appears to be much steeper than the computed gluon slopes because hadronization further softens the spectra as discussed later.

\section{Transport opacity dependence}

In this Section we provide a qualitative explanation for the remarkable invariance of the results on the actual angular dependence of the differential cross section (2). The reason that this simplification occurs is that for the $\mu$ and gluon energy range considered in these plots, the transport opacity is actually high enough that little memory of the initial gluon momentum direction remains after multiple scattering. Thus, isotropic scattering and $\mu=T$ forward scattering both lead to essentially a random reorientation of all the gluons involved.

In Fig. 12, aside from a small 10\% delta function component due to the gluons in the "corona" surface region that escape without rescattering, the bulk of the minijets undergo enough rescatterings that their final direction is 
randomized. In Fig. 13 we also show that the rapidity shift of gluons in each transverse momentum interval considered has the form close to one expected if local thermal equilibrium occurred:

$$
\begin{aligned}
\frac{d P}{d \Delta y} & =\int d m_{\perp i}^{2} d y_{i} d m_{\perp f}^{2} m_{\perp i} \cosh y_{i} m_{\perp f} \cosh \left(y_{i}+\Delta y\right) \\
& \times \frac{1}{4 T_{i}^{3}} \frac{1}{4 T_{f}^{3}} e^{-\left(m_{\perp i} \cosh y_{i}\right) / T_{i}} e^{-\left[m_{\perp f} \cosh \left(y_{i}+\Delta y\right)\right] / T_{f}} \\
& =\frac{\Delta y \cosh \Delta y-\sinh \Delta y}{\sinh ^{3} \Delta y} .
\end{aligned}
$$

The randomization of momenta is however not sufficient to ensure the validity of local equilibrium necessary for the applicability of nondissipative (Euler) hydrodynamics. This is proven by the dependence of the transverse momentum spectra and elliptic flow on the finite opacity parameter itself. In the hydrodynamic limit $\chi=\infty$ and the transport evolution is identical to the hydrodynamical evolution. However, we showed in detail in a previous study [17] that the solutions of the transport equation still differ very much from ideal hydrodynamics, for physically extreme $\chi \sim 20$ opacities. While no covariant $3+1 \mathrm{D}$ Navier-Stokes solutions are yet known, our transport solutions demonstrate the effects of dissipation through their dependence on $1 / \chi$.

The invariance of the transport solutions to the angular distributions for a fixed $\chi$ indicate however that we are not extremely far from the local thermal though dissipative limit. In particular, our high opacity solutions are far from the Eikonal (Knudsen) type dynamics as considered in [13].

A rough criterion for the validity of the Eikonal approximation is that the angle between the initial and final parton momenta in the laboratory frame satisfies $\Delta \theta \ll 1$, say $\Delta \theta<0.3$. For an energetic parton that undergoes $N$ elastic collisions, this angle can be approximated in an analogous way to random walk as

$$
\langle\Delta \theta\rangle_{N} \approx \sqrt{N\left\langle\Delta \theta^{2}\right\rangle_{N=1}} \sim \frac{\sqrt{s}}{2 E} \sqrt{N\left\langle\Delta \theta_{c m}^{2}\right\rangle}
$$

because the angles transform as $\Delta \theta \approx \Delta \theta_{c m} \sqrt{s} / 2 E$. In the small angle approximation, we can use Eq. (3) to estimate

$$
\left\langle\Delta \theta_{c m}^{2}\right\rangle \approx \frac{\sigma_{t}(s)}{\sigma_{0}} .
$$

Hence, for elastic collisions off typical thermal partons $(s \approx 6 E T, \mu=T)$, the Eikonal approximation is valid if $\sigma_{t}(s) / \sigma_{0}<E /(15 T N)$. For $N \sim 10$, this condition is satisfied for $E>20 T$. Note that the total cross section does not depend on the parton energy and therefore the number of collisions is approximately independent of energy. In Fig. 12 we see that the Eikonal limit is only approached slowly as the parton energy increases.

We conclude from these results that the pattern of "jet quenching", as observed at RHIC via the suppression of moderate transverse momentum particles and the saturation of elliptic flow above some critical $p_{\perp}$, can be reproduced if sufficiently high transport opacities are postulated with any angular distribution.

\section{HADRON SPECTRA}

The results in the previous Section pertain only to partons. To compare with experimental results, we must adopt a model of hadronization. Here we compute the hadronic observables from two different hadronization schemes.

\section{A. Hadronization via local parton-hadron duality}

The simplest hadronization scheme is based on the idea of local parton-hadron duality. If as in Ref. [21], we assume that each gluon gets converted to a pion with equal probability for the three isospin states, then we may approximate the transverse momentum distribution of negative charged hadrons roughly as

$$
f_{h^{-}}\left(\mathbf{p}_{\perp}\right) \approx f_{\pi^{-}}\left(\mathbf{p}_{\perp}\right)=\frac{1}{3} f_{g}\left(\mathbf{p}_{\perp}\right) .
$$

With the above prescription, elliptic flow does not change during hadronization, i.e., Figs. 4-8 show the negative hadron flow as well. Furthermore, the negative hadron $p_{\perp}$ spectra can be obtained from Figs. 9-11 via simply dividing by 3 . Consequently, the scaling (11) holds for the negative hadron flow and spectra as well.

In Fig. 7, the elliptic flow data by STAR are reproduced with a transport opacity $\chi_{b=0}=47.8$. For an initial gluon density $d N_{g}(0) / d \eta=1000$, this corresponds to $\sigma_{t} \approx 14 \mathrm{mb}$, i.e., to a total cross section of $\sigma_{0} \approx 45 \mathrm{mb}$ with $\mu=T_{0}$. If we took, on the other hand, the pQCD $g g$ cross section of $3 \mathrm{mb}$ with $\mu=T_{0}$, this opacity would correspond to an initial gluon density of $d N_{g}(0) / d \eta \sim 15000$ that is contradicted by the much smaller observed $d N_{c h} / d \eta \approx 600$.

The $p_{\perp}$ spectra provide a much stronger constraint on the initial gluon density as their absolute magnitude is proportional to it. At high opacities $(\chi>\sim 10)$, the need for high particle subdivisions poses a severe computational problem, therefore we can reliably compute particle spectra for semicentral collisions only. Nevertheless, the data measured by STAR in central collisions, where quenching due to parton energy loss is expected to be maximal, provides an important lower bound on the particle yields. Figure 11 shows that the elastic transport opacities $\chi<\sim 20$ considered here are compatible with this lower bound.

On the other hand, the cascade semicentral results are very much above the preliminary STAR spectra [30] for central collisions. The problem is that the fragmentation of quarks and gluons generally soften considerably the high- $p_{\perp}$ spectra as we show in the next Section. 


\section{B. Hadronization via independent fragmentation}

The next simplest hadronization scheme is the fragmentation of gluons as independent jets. We consider here only the $g \rightarrow \pi^{ \pm}$channel with the next-to-leadingorder fragmentation function taken from Ref. [28]. We took the scale factor $s \equiv \log \left(Q^{2}\right) / \log \left(Q_{0}^{2}\right)$ to be zero because the initial HIJING gluon distribution is already "self-quenched" due to initial and final state radiation. Also, since we do not consider the contribution of low- $p_{\perp}$ soft multiparticle production (beam jet fragmentation), we consider from now on only hadrons with $p_{\perp}>2 \mathrm{GeV}$.

Figure 14 shows the final impact-parameter-averaged negative hadron flow as a function of the transport opacity. The flow pattern and the magnitude of the flow are much the same as for partons in Fig. 7. Hence, we get the same constraint on the initial parameters as for hadronization via local parton-hadron duality. In the $p_{\perp}<2 \mathrm{GeV}$ region this simple calculation does not reproduce the data because it does not include contributions coming from soft physics.

The $p_{\perp}$ spectra of charged hadrons are shown as a function of transport opacity and impact parameter in Figs. 15 and 16. In addition to quenching because of energy loss, the final pion spectra are further quenched due to independent fragmentation. With this additional quenching, the parton cascade results approach the preliminary STAR data [30], as indicated in Fig. 17, only for rather extreme $\sigma_{0}(\mu=T) \approx 100 \mathrm{mb}$ if HIJING $d N_{g} / d y=210$ is assumed, or if $\sigma_{0} \approx 25 \mathrm{mb}$ and EKRT $d N_{g} / d y=1000$ is assumed. These elastic cross sections exceed the conventional few mb pQCD cross sections at this scale by at least an order of magnitude.

\section{CONCLUSIONS}

The MPC parton cascade technique was applied to solve the covariant Boltzmann transport numerically and compute new observables at RHIC. Our focus was on the preliminary differential elliptic flow and charged hadron moderate $p_{\perp}>2 \mathrm{GeV} / \mathrm{c}$ spectra. We compared results using two different hadronization schemes: independent fragmentation and local parton-hadron duality.

Our main result is that if only elastic scattering is taken into account in the covariant Boltzmann equation, extremely large densities and/or elastic parton cross sections, $\sigma_{t o t} d N / d \eta \sim 80$ times the HIJING estimate, are needed to reproduce the elliptic flow data [16]. Hadronization via local parton-hadron duality fails to reproduce the rapidly falling high- $p_{\perp}$ spectra. However, independent fragmentation of our MPC solutions compare well to the charged hadron $p_{\perp}$ spectra when rather large elastic transport opacities are postulated.

The solutions clearly demonstrate how finite (even extreme) reaction rates in $A+A$ lead to major deviations from ideal hydrodynamic transverse flow effects at transverse momentum $p_{T}>2 \mathrm{GeV}$. The pattern of quenching found with MPC is surprisingly similar to that obtained in the two component model of GVW [13]. The main difference between the high opacity MPC solutions reported here and the low opacity results of GVW is that the latter include radiative energy loss in an Eikonal formalism joined to a parametrized phenomenological "hydrodynamic" component.

It is known that radiative energy loss of ultrarelativistic partons is much larger than elastic energy loss in a medium for a fixed cross section. In GVW a similar quenching pattern was obtained with more modest initial densities $d N_{g} / d y \sim 500$ and small pQCD elastic rates because the induced gluon radiation associated with multiple elastic collisions is large enough to compensate for the small elastic transport opacity in that case. In MPC the same level of quenching is achieved only when the elastic opacity is increased artificially by an order of magnitude. Therefore, the present study confirms the expectation that elastic scattering alone is not enough to generate the degree of collectivity observed now at RHIC.

\section{OUTLOOK}

The results presented here underscore the urgent need to develop practical convergent algorithms to incorporate inelastic $2 \leftrightarrow 3$ processes. Preliminary work in Ref. [18] indicated a rather slow convergence towards Lorentz covariance using the particle subdivision technique. Unlike the $\ell^{-1 / 2}$ convergence of $2 \rightarrow 2$ transport solutions, a much slower rate of convergence $\propto \ell^{-1 / 5}$ is expected with the parton subdivision method used to retain Lorentz covariance of $2 \leftrightarrow 3$ processes.

In addition, a more powerful covariant approximation to Boltzmann transport theory may be needed to overcome the overwhelming computational difficulty in the high opacity regime for central collisions. We found that even for the case of elastic scattering, particle subdivisions up to 1000 are required to maintain covariance and stabilize the final spectra.

Finally, we note that all results in this paper pertain to slowly varying, smooth initial conditions. In Ref. [29], it was suggested that copious minijet production may induce large (nonstatistical) local fluctuations that could evolve in a turbulent manner. A transport study of differential elliptic flow and jet quenching in such inhomogeneous initial conditions would be interesting to compare to the presently known hydrodynamic and Boltzmann solutions.

\section{ACKNOWLEDGMENTS}

We acknowledge the Parallel Distributed Systems Facility at the National Energy Research Scientific Comput- 
ing Center for providing computing resources. We also thank RMKI/KFKI for hospitality during the completion of this work.

This work was supported by the Director, Office of Energy Research, Division of Nuclear Physics of the Office of High Energy and Nuclear Physics of the U.S. Department of Energy under contract No. DE-FG-02-93ER40764. M. G. also was partially supported by Collegium Budapest.

\section{APPENDIX: COMPARISON OF ELLIPTIC FLOW DEFINITIONS}

The main reason that definition (12) gives a very similar elliptic flow to definition (13), contrary to the opposite observation from hydrodynamics, is the difference between the hydro and the cascade $v_{2}(b)$ shapes. Hydrodynamical models predict an increasing $v_{2}$ out to $b_{m} \approx 12-13 \mathrm{fm}$, while the cascade $v_{2}$ peaks at $b_{m} \approx 8$ $\mathrm{fm}$. The $b<b_{m}$ region where $v_{2}$ is small gets a larger weight in the STAR definition, while the $b>b_{m}$ region where $v_{2}$ is as well small gets a smaller weight. The former effect tends to reduce elliptic flow, while the latter tends to increase it. In the case of hydrodynamics, the first effect dominates, while for the cascade, the second effect turns out to be larger.

We illustrate this with a simple analytic calculation. The impact parameter dependence of elliptic flow can be fitted with the general form

$$
v_{2}(b)=K\left(\frac{b}{B}\right)^{c}\left(1-\frac{b}{B}\right)^{d},
$$

while the particle spectrum is approximately linear in the $b=2-12 \mathrm{fm}$ region of interest

$$
\frac{d N}{d y d p_{\perp}}(b)=C\left(1-\frac{b}{B^{\prime}}\right) .
$$

Here the parameters $K, B, B^{\prime}, c, d$, and $C$ in general depend on $p_{\perp}$.

It is easy to show that for the above functions definition (12) gives an elliptic flow $v_{2}^{a v}=2 K \Gamma(c+2) \Gamma(d+1) / \Gamma(c+$ $d+3)$, while definition $(13)$ yields $v_{2}^{S T A R}=6 K \Gamma(c+$ 2) $\Gamma(d+2) / \Gamma(c+d+4)$, provided we assume $B=B^{\prime}$ and integrate up to $b_{\max }=B$ in both cases. Therefore,

$$
\frac{v_{2}^{S T A R}}{v_{2}^{a v}}=\frac{3 d+3}{c+d+3},
$$

which is larger than one, if and only if $2 d>c$. For our cascade results, the fits to $v_{2}(b)$ give $d / c \sim 0.6-1.4$ $\left[c\left(p_{\perp}\right) \sim 1.1-3.3, d\left(p_{\perp}\right) \sim 1.0-4.5\right]$, therefore we have $v_{2}^{S T A R}\left(p_{\perp}\right)>v_{2}^{a v}\left(p_{\perp}\right)$. On the other hand, hydrodynamics gives an approximately linear $v_{2}(b)$, i.e., $c=1$ and $d=0$, which results in $v_{2}^{S T A R}\left(p_{\perp}\right)<v_{2}^{a v}\left(p_{\perp}\right)$.

We found essentially the same when taking an exponential fit to $d N / d p_{\perp}(b)$ instead of a linear one.
Equation (A3) yields $v_{2}^{S T A R} / v_{2}^{a v} \sim 1.1-1.5$ for the cascade (depending on $p_{\perp}$ and initial conditions). However, in reality $B^{\prime}$ is smaller than $B$ (typically $B^{\prime} \approx 11-13$ $\mathrm{fm}$, while $B \approx 13-16 \mathrm{fm}$ ), which influences $v_{2}^{S T A R}$. Furthermore, the upper limit of integration $b_{\max }=12 \mathrm{fm}$ is also smaller than $B$. This affects primarily $v_{2}^{a v}$, through the normalization constant $1 / b_{\max }^{2}$. The integrals in both definitions are to a large degree insensitive to variations of $b_{\max }$ because the integrands cut off naturally at large $b$.

To illustrate these effects, we repeat the previous analytic calculation with $b_{\max } \equiv x B$ for $v_{2}^{a v}$, while $B^{\prime} \equiv y B$ and $b_{\max } \equiv x^{\prime} B^{\prime}$ for $v_{2}^{S T A R}$. The integrals yield

$$
\begin{aligned}
& v_{2}^{S T A R}\left(x^{\prime}, y\right)=6 K \frac{y B_{y x^{\prime}}(c+2, d+1)-B_{y x^{\prime}}(c+3, d+1)}{y^{3} x^{\prime 2}\left(3-2 x^{\prime}\right)} \\
& v_{2}^{a v}(x)=2 K \frac{B_{x}(c+2, d+1)}{x^{2}},
\end{aligned}
$$

where $B_{z}(a, b) \equiv \int_{0}^{z} d t t^{a-1}(1-t)^{b-1}$ is the incomplete beta function. Thus,

$$
\begin{aligned}
& \frac{v_{2}^{S T A R}\left(1-x^{\prime}, 1-y\right)}{v_{2}^{S T A R}(1,1)}=1+\frac{2 d-c}{d+1} y+O\left(x^{2}\right)+O\left(y^{2}\right) \\
& \frac{v_{2}^{a v}(1-x)}{v_{2}^{a v}(1)}=1+2 x+O\left(x^{\min \{2, d+1\}}\right),
\end{aligned}
$$

i.e., the leading correction to $v_{2}^{a v}$ comes at first order in $\left(b_{\max }-B\right)$, while the leading correction to $v_{2}^{S T A R}$ comes at first order in $\left(B^{\prime}-B\right)$.

For parameters and initial conditions considered in this study, the corrected formulas (A4) yield $v_{2}^{S T A R} / v_{2}^{a v} \sim$ $0.9-1.05$ (with $x^{\prime}=1$ ). The uncertainty of $v_{2}^{S T A R}$ due to the unknown experimental cutoff $b_{\max }$ (i.e., $x^{\prime}$ ) is less than $5 \%$. For the hydro $(c=1, d=0)$, this analysis gives a much smaller ratio $v_{2}^{S T A R} / v_{2}^{a v}=3\left(1-x^{\prime}\right)(1-y) / 4(1-$ $x) \approx 0.75$.

[1] H. Stocker and W. Greiner, Phys. Rept. 137, 277 (1986)

[2] J. Ollitrault, Phys. Rev. D 46, 229 (1992).

[3] H. Sorge, Phys. Rev. Lett. 78, 2309 (1997) [nuclth/9610026];

[4] H. Sorge, Nucl. Phys. A661, 577 (1999) [nuclth/9906051].

[5] S. Voloshin and Y. Zhang, Z. Phys. C 70, 665 (1996) [hepph/9407282]; A. M. Poskanzer and S. A. Voloshin, Phys. Rev. C 58, 1671 (1998) [nucl-ex/9805001]; S. A. Voloshin and A. M. Poskanzer, Phys. Lett. B474, 27 (2000) [nuclth/9906075].

[6] P. F. Kolb, U. Heinz, P. Huovinen, K. J. Eskola and K. Tuominen, hep-ph/0103234; P. Huovinen, P. F. Kolb, U. Heinz, P. V. Ruuskanen and S. A. Voloshin, Phys. Lett. B 503, 58 (2001) [hep-ph/0101136]; P. F. Kolb, 
P. Huovinen, U. Heinz and H. Heiselberg, Phys. Lett. B

PUB-2001-011 500, 232 (2001) [hep-ph/0012137]; P. F. Kolb, J. Sollfrank and U. Heinz, Phys. Lett. B 459, 667 (1999) [nuclth/9906003].

[7] L. P. Csernai and D. Rohrich, Phys. Lett. B 458, 454 (1999) [nucl-th/9908034].

[8] D. Teaney, J. Lauret and E. V. Shuryak, nuclth/0011058.

[9] B. Zhang, M. Gyulassy and C. M. Ko, Phys. Lett. B455, 45 (1999) [nucl-th/9902016].

[10] E. E. Zabrodin, C. Fuchs, L. V. Bravina and A. Faessler, Phys. Rev. C63, 034902 (2001) [nucl-th/0006056], E. E. Zabrodin et al., nucl-th/0104054.

[11] M. Bleicher and H. Stocker, hep-ph/0006147.

[12] X. Wang, Phys. Rev. C 63, 054902 (2001) [nuclth/0009019].

[13] M. Gyulassy, I. Vitev and X. N. Wang, Phys. Rev. Lett. 86, 2537 (2001), [nucl-th/0012092].

[14] K. H. Ackermann et al. [STAR Collaboration], Phys. Rev. Lett. 86, 402 (2001) [nucl-ex/0009011].

[15] H. Appelshauser et al. [NA49 Collaboration], Phys. Rev. Lett. 80, 4136 (1998) [nucl-ex/9711001].

[16] R.J. Snellings [STAR Collaboration], QM2001, nuclex/0104006.

[17] D. Molnar and M. Gyulassy, Phys. Rev. C 62, 054907 (2000) [nucl-th/0005051].

[18] D. Molnár, Nucl. Phys. A661, 236c (1999) [nuclth/9907090].

[19] D. Molnar and M. Gyulassy, nucl-th/0102031; D. Molnar and M. Gyulassy, Nucl. Phys. A in press, nuclth/0104018.

[20] M. Gyulassy and X. Wang, Comput. Phys. Commun. 83, (1994) 307 [nucl-th/9502021].

[21] K. J. Eskola, K. Kajantie, P. V. Ruuskanen, and K. Tuominen, Nucl. Phys. B570, 379 (2000) [hep$\mathrm{ph} / 9909456]$.

[22] D. Molnár, MPC 1.0.6. This parton cascade code used in the present study can be downloaded from WWW at http://nt3.phys.columbia.edu/people/molnard.

[23] B. Zhang, Comput. Phys. Commun. 109, 193 (1998) [nucl-th/9709009].

[24] M. Gyulassy, Y. Pang, and B. Zhang, Nucl. Phys. A626, (1997) 999.

[25] B. Zhang, M. Gyulassy, and Y. Pang, Phys. Rev. C 58, (1998) 1175 [nucl-th/9801037].

[26] Y. Pang, RHIC 96 Summer Study, CU-TP-815 preprint (unpublished); Generic Cascade Program (GCP) documentation available at WWW site http://rhic.phys.columbia.edu/rhic/gcp.

[27] Proc. of Open Standards for Cascade Models for RHIC (OSCAR), BNL-64912, June 23-27, 1997, eds. M. Gyulassy and Y. Pang; Source codes and documentation for transport models under the OSCAR standard can be downloaded from the OSCAR WWW site http://rhic.phys.columbia.edu/rhic/.

[28] J. Binnewies, B. A. Kniehl and G. Kramer, Z. Phys. C 65, 471 (1995) [hep-ph/9407347].

[29] M. Gyulassy, D. Rischke, and B. Zhang, Nucl. Phys. A613 (1997) 397.

[30] J. C. Dunlop [STAR Collaboration], CERN-ALICE- 


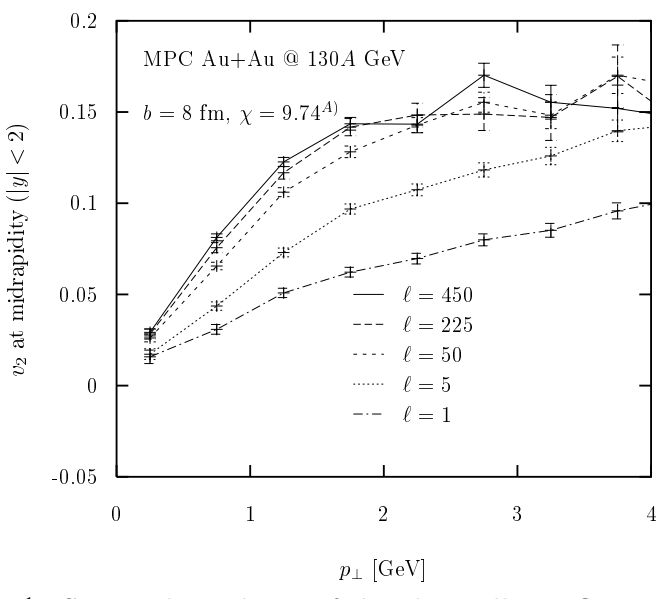

FIG. 1. Strong dependence of the gluon elliptic flow on parton subdivision as a function of $p_{\perp}$ is shown for $\mathrm{Au}+\mathrm{Au}$ at $\sqrt{s}=130 \mathrm{~A}$ $\mathrm{GeV}$ with $b=8 \mathrm{fm}$. Solutions for transport opacity $\chi=9.74^{A}$ ) (see Table I), and particle subdivisions $\ell=1,5,50,225$, and 450 are shown.

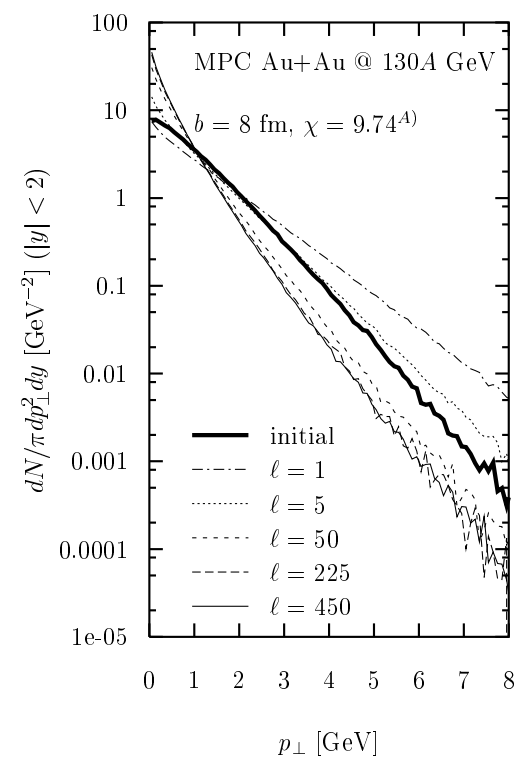

FIG. 2. Strong dependence of the final gluon $p_{\perp}$ spectra on parton subdivision is shown for $\mathrm{Au}+\mathrm{Au}$ at $\sqrt{s}=130 \mathrm{~A} \mathrm{GeV}$ with $b=8 \mathrm{fm}$. Solutions for transport opacity $\chi=9.74^{A}$ ) (see Table I), and particle subdivisions $\ell=1,5,50,225$, and 450 are shown as in Fig. 1. The spectra are normalized here to $d N(0) / d \eta=210$.
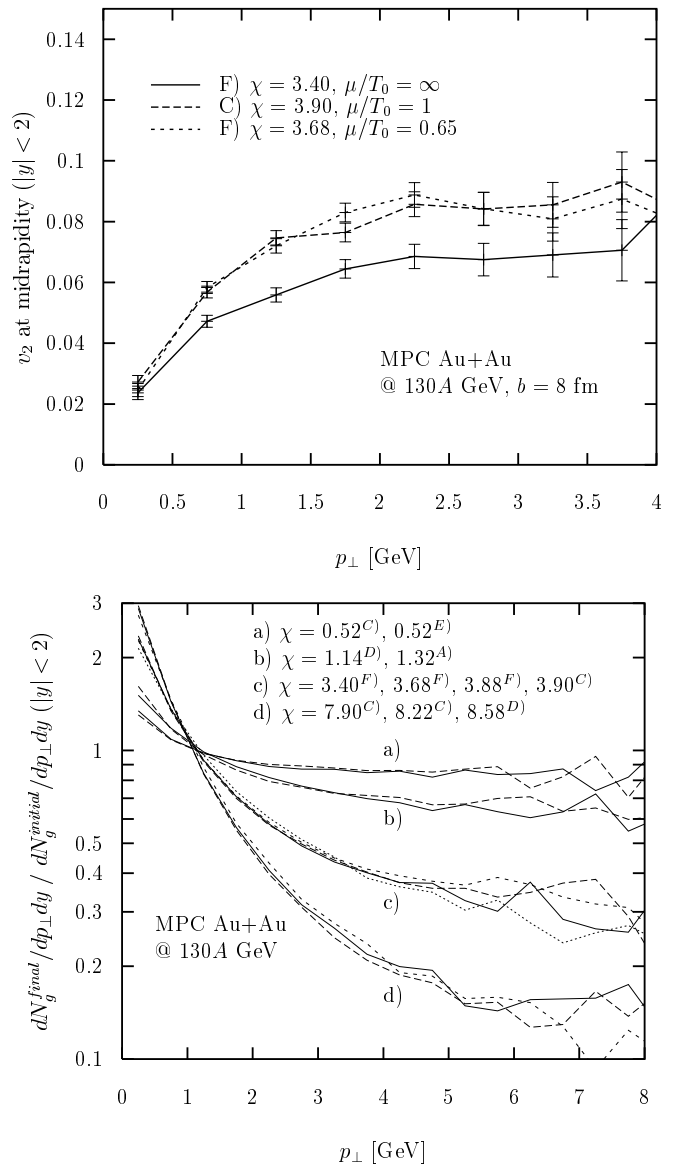

FIG. 3. The very weak dependence of the elliptic flow and gluon spectra quenching on the angular distribution of the parton cross section is shown. See Table I for the simulation parameters corresponding to each curve. The solutions are seen to depend mainly on the transport opacity.

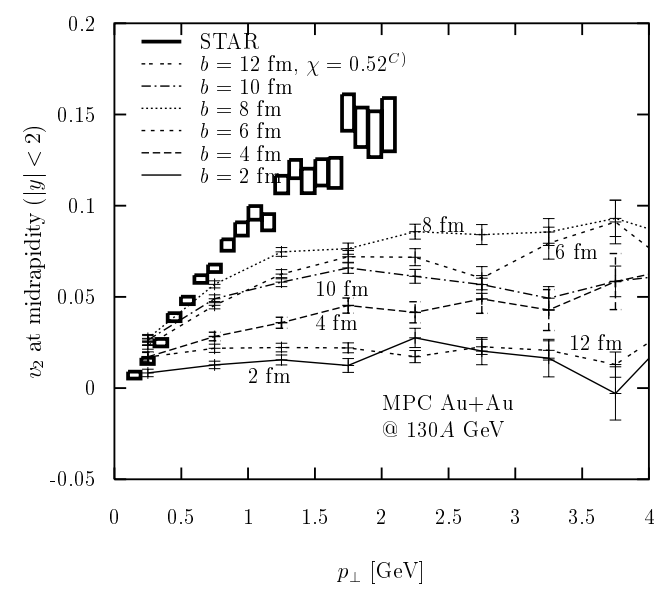

FIG. 4. Gluon elliptic flow as a function of $p_{\perp}$ for $\mathrm{Au}+\mathrm{Au}$ at $\sqrt{s}=130 A \mathrm{GeV}$ with impact parameters $b=2,4,6,8,10$, and $12 \mathrm{fm}$ is shown for transport opacities $\chi=7.90^{C)}, 6.98^{C)}, 5.68^{C}$ ), $3.90^{C)}, 1.98^{C)}$, and $0.52^{C)}$. STAR data [14] below $2 \mathrm{GeV} / c$ are shown. Preliminary STAR data [16] suggest that $v_{2} \sim 0.15-0.17$ may saturate in the $2<p_{T}<4 \mathrm{GeV} / c$ range. 


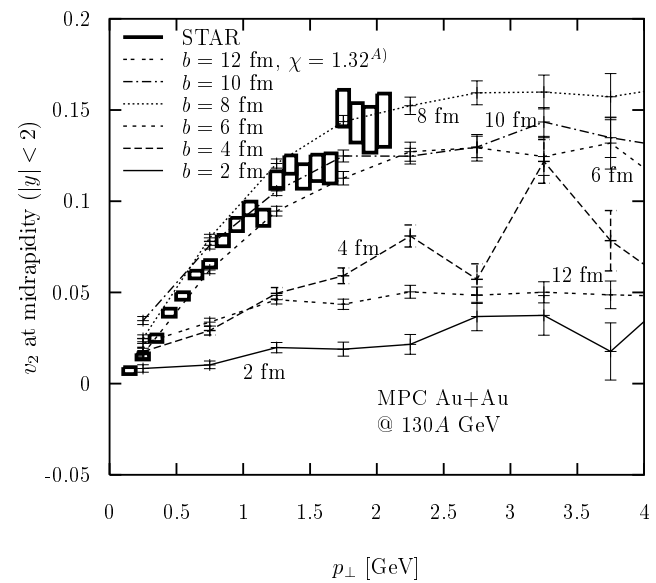

FIG. 5. Same as Fig. 4 except that solutions for transport opacities $\chi=19.4^{A)}, 17.2^{A)}, 14.1^{A)}, 9.74^{A)}, 5.00^{A)}$, and $1.32^{A)}$ are shown. STAR data [14] below $2 \mathrm{GeV} / c$ are shown. Preliminary STAR data [16] suggest that $v_{2} \sim 0.15-0.17$ may saturate in the $2<p_{T}<4 \mathrm{GeV} / c$ range.

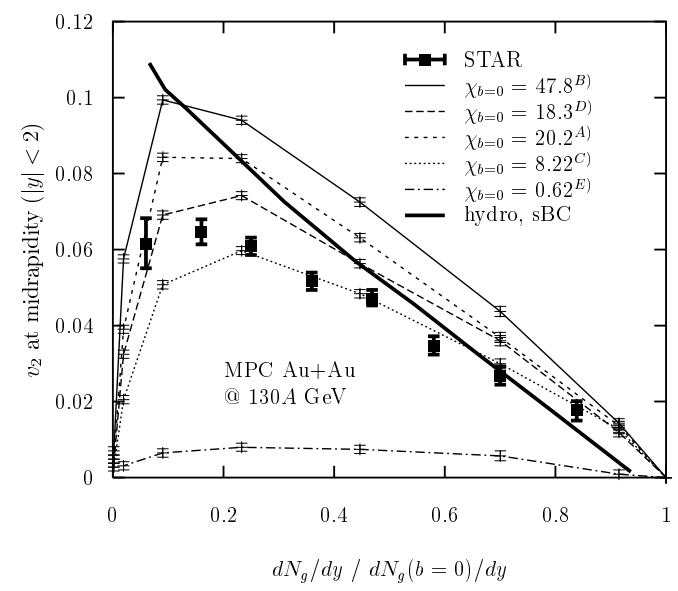

FIG. 6. Gluon elliptic flow as a function of centrality for $\mathrm{Au}+\mathrm{Au}$ at $\sqrt{s}=130 A \mathrm{GeV}$ is shown for transport opacities $\chi_{b=0}=0.62^{E)}$, $8.22^{C)}, 18.3^{D)}, 20.2^{A}$ ) and $\left.47.8^{B}\right)$ for $b=0$. Identical to the charged hadron elliptic flow as a function of $n_{c h} / n_{c h}^{\max }$, if the gluons are hadronized via local parton-hadron duality. The ideal hydrodynamics result is taken from [6] with the so called sBC initial conditions. STAR data [14] are also shown.

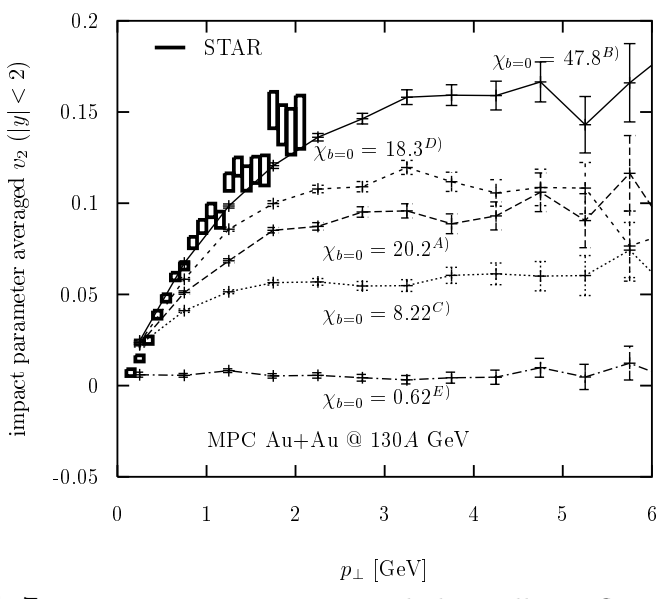

FIG. 7. Impact parameter averaged gluon elliptic flow is shown as a function of $p_{\perp}$ for $\mathrm{Au}+\mathrm{Au}$ at $\sqrt{s}=130 \mathrm{~A} \mathrm{GeV}$ with transport opacities $\chi_{b=0}=0.62^{E)}, 8.22^{C)}, 18.3^{D)}, 20.2^{A)}$, and $47.8^{B}$ ) for $b=0$. Identical to the charged hadron elliptic flow if the gluons are hadronized via local parton-hadron duality. STAR data [14] below $2 \mathrm{GeV} / c$ are shown. Preliminary STAR data [16] suggest that $v_{2} \sim 0.15-0.17$ may saturate in the $2<p_{T}<4 \mathrm{GeV} / c$ range.

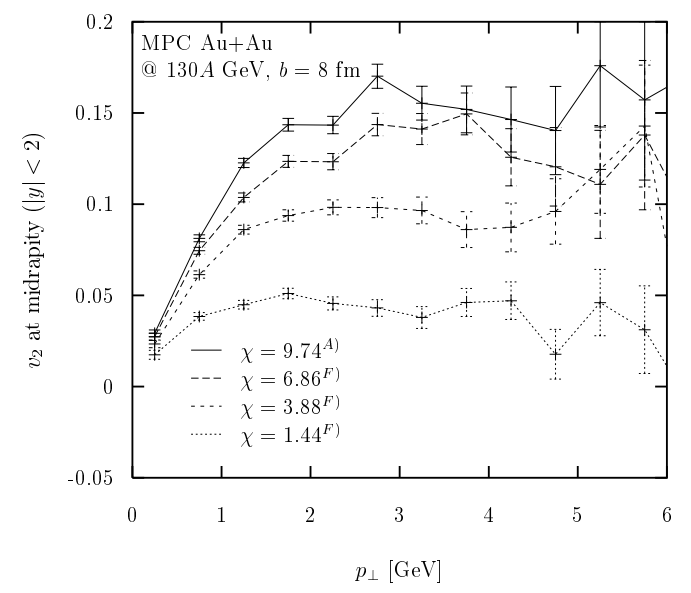

FIG. 8. Gluon elliptic flow as a function of $p_{\perp}$ is shown for $\mathrm{Au}+\mathrm{Au}$ at $\sqrt{s}=130 \mathrm{~A} \mathrm{GeV}$ with $b=8 \mathrm{fm}$ and $\mu / T_{0}=0.226,0.45$, 0.71 , and 1 (transport opacities $\chi=1.44^{F)}, 3.88^{F)}, 6.86^{F)}$, and $\left.9.74^{A)}\right)$. 

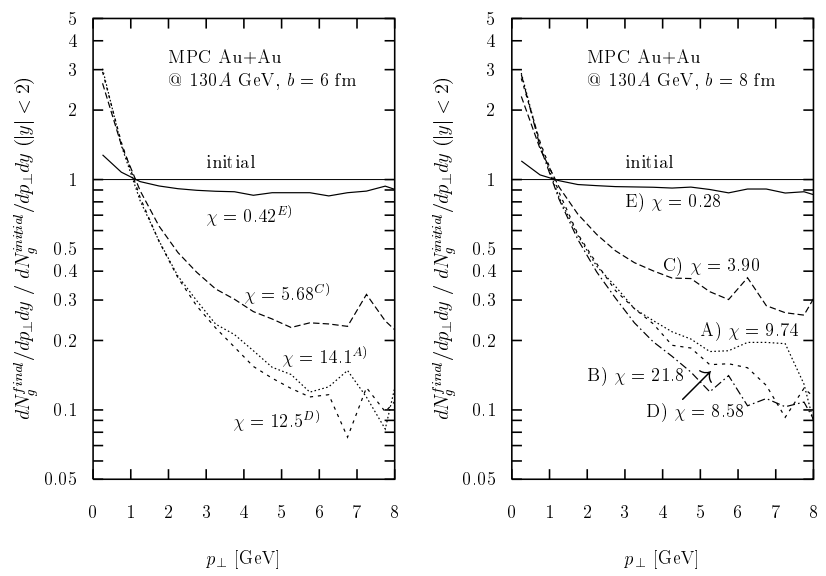

FIG. 9. Final gluon $p_{\perp}$ spectra relative to the thermal initial spectrum are shown as a function of transport opacity for $\mathrm{Au}+\mathrm{Au}$ at $\sqrt{s}=130 A \mathrm{GeV}$ with $b=6 \mathrm{fm}$ (left) and $b=8 \mathrm{fm}$ (right). Proportional to the charged hadron $p_{\perp}$ spectra if the gluons are hadronized via local parton-hadron duality.
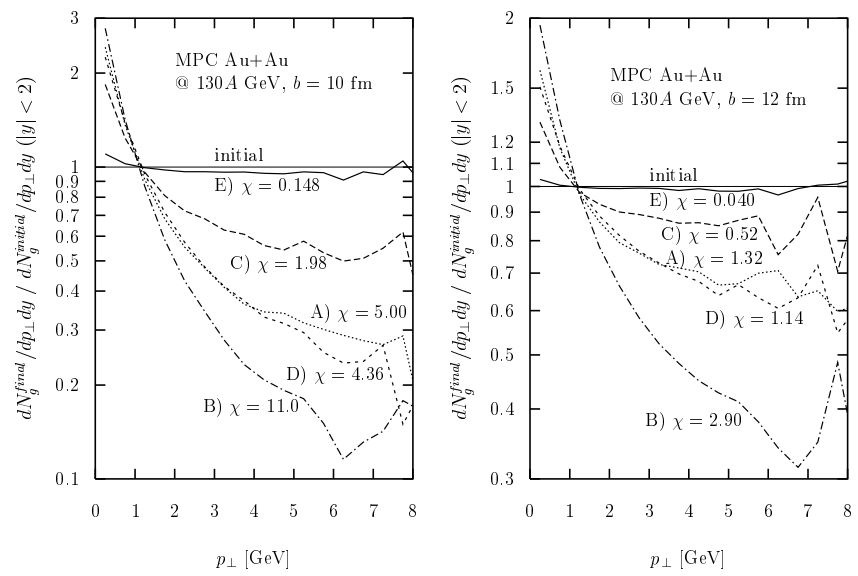

FIG. 10. Final gluon $p_{\perp}$ spectra relative to the thermal initial spectrum are shown as a function of transport opacity for $\mathrm{Au}+\mathrm{Au}$ at $\sqrt{s}=130 A \mathrm{GeV}$ with $b=10 \mathrm{fm}$ (left) and $b=12 \mathrm{fm}$ (right). Proportional to the charged hadron $p_{\perp}$ spectra if the gluons are hadronized via local parton-hadron duality.

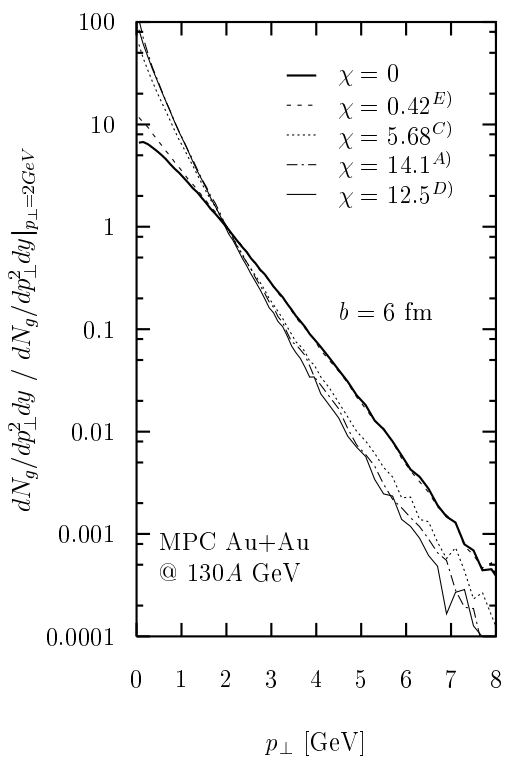

FIG. 11. Final gluon $p_{\perp}$ spectra as a function of transport opacity are shown for $\mathrm{Au}+\mathrm{Au}$ at $\sqrt{s}=130 A \mathrm{GeV}$ with $b=6 \mathrm{fm}$ and with all curves normalized to 1 at $p_{\perp}=2 \mathrm{GeV}$. This shows the quenching at high $p_{\perp}$ relative to $p_{\perp}=2 \mathrm{GeV}$. Also proportional to the charged hadron $p_{\perp}$ spectra if the gluons are hadronized via local parton-hadron duality. Preliminary STAR data [30] suggest that this ratio may reach $10^{-4}$ in the $p_{\perp} \sim 5-6 \mathrm{GeV} / \mathrm{c}$ range.

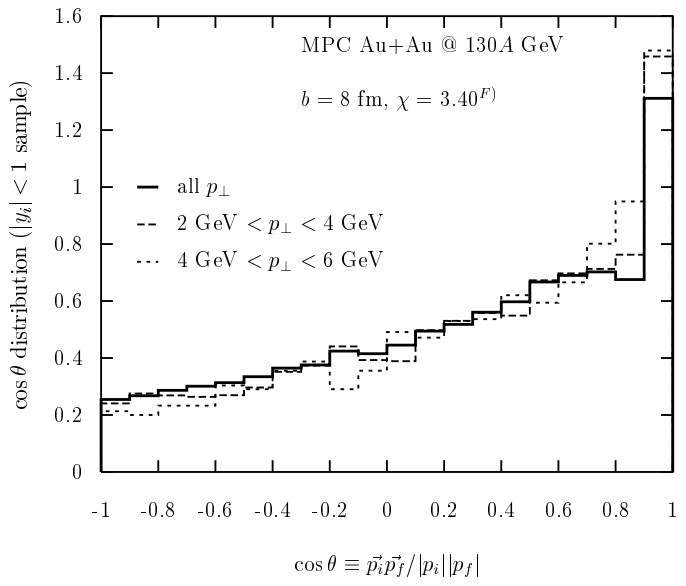

FIG. 12. Correlation between initial and final gluon momentum direction is shown for $\mathrm{Au}+\mathrm{Au}$ at $\sqrt{s}=130 A \mathrm{GeV}$ with $b=8 \mathrm{fm}$ and transport opacity $\chi=3.40^{F)}$. 


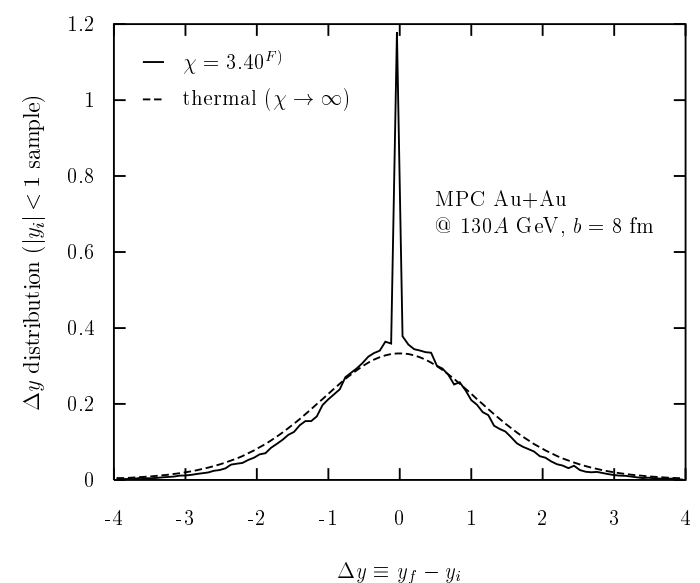

FIG. 13. Correlation between initial and final gluon rapidity is shown for $\mathrm{Au}+\mathrm{Au}$ at $\sqrt{s}=130 \mathrm{~A} \mathrm{GeV}$ with $b=8 \mathrm{fm}$ and transport opacity $\chi=3.40^{F)}$.

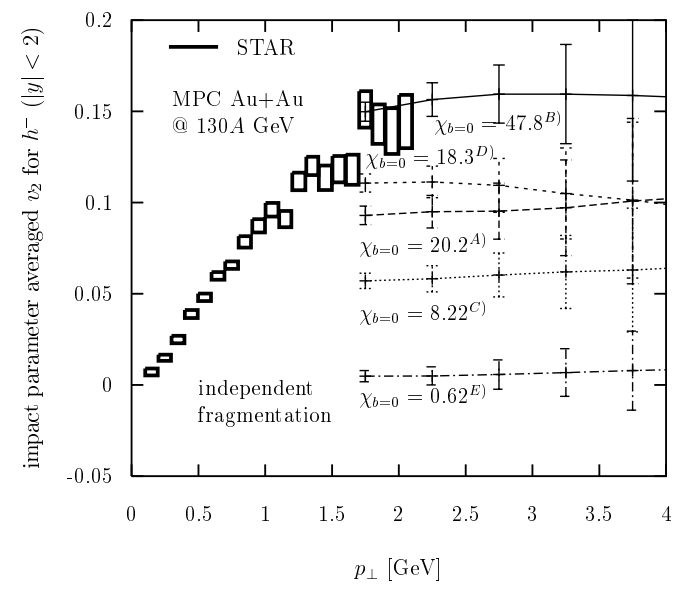

FIG. 14. Impact parameter averaged negative hadron elliptic flow as a function of $p_{\perp}$ is shown for $\mathrm{Au}+\mathrm{Au}$ at $\sqrt{s}=130 \mathrm{~A} \mathrm{GeV}$ with transport opacities $\chi_{b=0}=0.62^{E)}, 8.22^{C}, 18.3^{D)}, 20.2^{A)}$, and $47.8^{B)}$ at $b=0$ and hadronization via independent fragmentation. The $p_{\perp}<2 \mathrm{GeV}$ region is not plotted because it is dominated by soft contributions not addressable via $\mathrm{pQCD}$ jet fragmentation physics. STAR data [14] below $2 \mathrm{GeV} / c$ are shown. Preliminary STAR data [16] suggest that $v_{2} \sim 0.15-0.17$ may saturate in the $2<p_{T}<4 \mathrm{GeV} / c$ range.
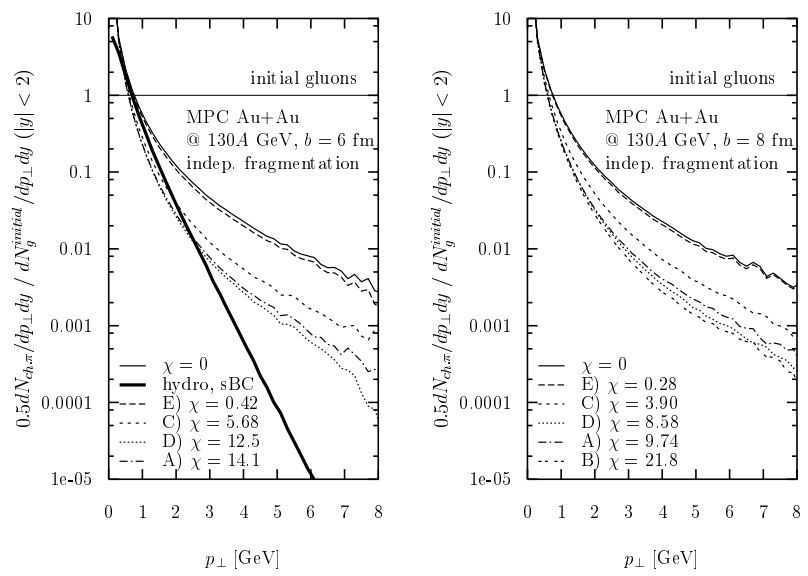

FIG. 15. Final negative hadron $p_{\perp}$ spectra via independent fragmentation are shown relative to the thermal initial gluon spectrum for $\mathrm{Au}+\mathrm{Au}$ at $\sqrt{s}=130 A \mathrm{GeV}$ with $b=6 \mathrm{fm}$ (left) and $b=8 \mathrm{fm}$ (right). The ideal hydrodynamics result in the left figure is taken from [6] with the so called sBC initial conditions. It was extrapolated beyond $p_{\perp}=3 \mathrm{GeV}$ using an exponential fit to the $d N / p_{\perp} d p_{\perp}$ distribution between 2 and $3 \mathrm{GeV}$.
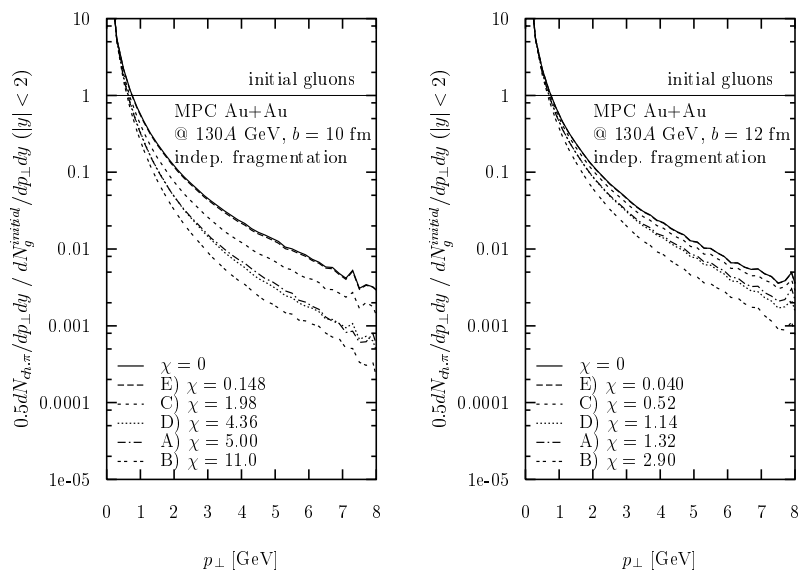

FIG. 16. Final negative hadron $p_{\perp}$ spectra via independent fragmentation are shown relative to the thermal initial gluon spectrum for $\mathrm{Au}+\mathrm{Au}$ at $\sqrt{s}=130 \mathrm{~A} \mathrm{GeV}$ with $b=10 \mathrm{fm}$ (left) and $b=12 \mathrm{fm}$ (right). 


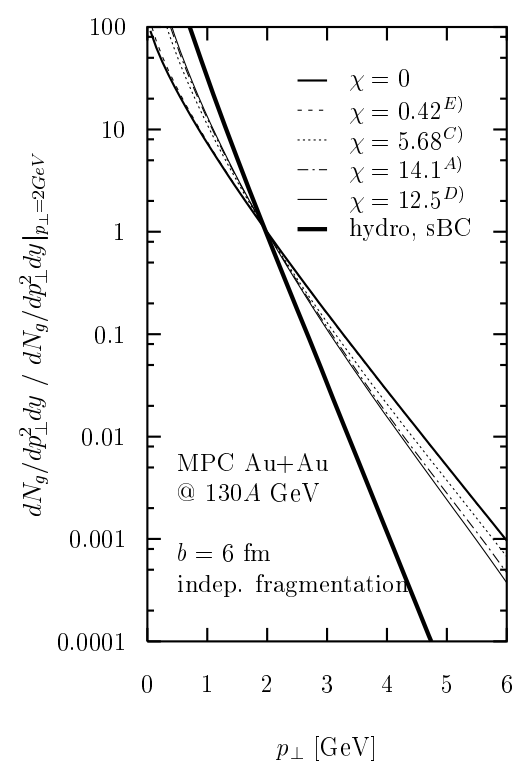

FIG. 17. Final negative hadron $p_{\perp}$ spectra via independent fragmentation are shown relative to the thermal initial gluon spectrum for $\mathrm{Au}+\mathrm{Au}$ at $\sqrt{s}=130 \mathrm{~A} \mathrm{GeV}$ with $b=6 \mathrm{fm}$ and with all curves normalized to 1 at $p_{\perp}=2 \mathrm{GeV}$. This shows the quenching at high $p_{\perp}$ relative to $p_{\perp}=2 \mathrm{GeV}$. The ideal hydrodynamics result is taken from [6] with the so called sBC initial conditions, and was extrapolated beyond $p_{\perp}=3 \mathrm{GeV}$ using an exponential fit to the $d N / p_{\perp} d p_{\perp}$ distribution between 2 and $3 \mathrm{GeV}$. Preliminary STAR data [30] suggest that this ratio may reach $10^{-4}$ in the $p_{\perp} \sim 5-6$ $\mathrm{GeV} / \mathrm{c}$ range.

\begin{tabular}{|c|c|c||c|c|c|}
\hline \hline \multicolumn{2}{|c||}{$\mathbf{A}) \sigma_{0}=100 \mathrm{mb}, T_{0} / \mu=1$} & \multicolumn{3}{|c|}{ B) $\sigma_{0}=100 \mathrm{mb}, T_{0} / \mu=0$} \\
\hline$b[\mathrm{fm}]$ & $\langle n\rangle$ & $\chi$ & $b[\mathrm{fm}]$ & $\langle n\rangle$ & $\chi$ \\
\hline \hline 0 & 33.0 & 20.2 & 0 & 35.8 & 47.8 \\
\hline 2 & 31.7 & 19.4 & 2 & 34.3 & 45.8 \\
\hline 4 & 28.1 & 17.2 & 4 & 30.2 & 40.2 \\
\hline 6 & 23.0 & 14.1 & 6 & 24.0 & 32.0 \\
\hline 8 & 15.9 & 9.74 & 8 & 16.3 & 21.8 \\
\hline 10 & 8.16 & 5.00 & 10 & 8.23 & 11.0 \\
\hline 12 & 2.15 & 1.32 & 12 & 2.18 & 2.90 \\
\hline \hline
\end{tabular}

\begin{tabular}{|l||l|}
\hline C) $\sigma_{0}=40 \mathrm{mb}, T_{0} / \mu=1$ & D) $\sigma_{0}=40 \mathrm{mb}, T_{0} / \mu=0$
\end{tabular}

\begin{tabular}{|c|c|c||c|c|c|}
\hline$b[\mathrm{fm}]$ & $\langle n\rangle$ & $\chi$ & $b[\mathrm{fm}]$ & $\langle n\rangle$ & $\chi$ \\
\hline \hline 0 & 13.4 & 8.22 & 0 & 13.7 & 18.3 \\
\hline 2 & 12.9 & 7.90 & 2 & 13.2 & 17.6 \\
\hline 4 & 11.4 & 6.98 & 4 & 11.6 & 15.5 \\
\hline 6 & 9.26 & 5.68 & 6 & 9.38 & 12.5 \\
\hline 8 & 6.37 & 3.90 & 8 & 6.44 & 8.58 \\
\hline 10 & 3.23 & 1.98 & 10 & 3.27 & 4.36 \\
\hline 12 & 0.86 & 0.52 & 12 & 0.86 & 1.14 \\
\hline
\end{tabular}

\begin{tabular}{|c|c|c||c|c|c|c|}
\hline \hline $\mathbf{E}) \sigma_{0}=3 \mathrm{mb}, T_{0} / \mu=1$ & \multicolumn{1}{||}{ F) various, $b=8 \mathrm{fm}$} \\
\hline$b[\mathrm{fm}]$ & $\langle n\rangle$ & $\chi$ & $\sigma_{0}[\mathrm{fm}]$ & $T_{0} / \mu$ & $\langle n\rangle$ & $\chi$ \\
\hline \hline 0 & 1.00 & 0.62 & 60 & 1.54 & 9.51 & 3.68 \\
\hline 2 & 0.96 & 0.58 & 16 & 0 & 2.55 & 3.40 \\
\hline 4 & 0.85 & 0.52 & 100 & 1.40 & 15.9 & 6.86 \\
\hline 6 & 0.69 & 0.42 & 100 & 2.21 & 15.7 & 3.88 \\
\hline 8 & 0.47 & 0.28 & 100 & 4.43 & 15.5 & 1.44 \\
\hline 10 & 0.24 & 0.148 & & & & \\
\hline 12 & 0.064 & 0.040 & & & & \\
\hline \hline
\end{tabular}

TABLE I. Parameters and transport opacity for each transport solution computed via MPC for the present study. 